\title{
OPEN Drug repurposing for coronavirus (SARS-CoV-2) based on gene co-expression network analysis
}

\author{
Habib MotieGhader ${ }^{1,2}$, Esmaeil Safavi ${ }^{1,3}$, Ali Rezapour ${ }^{4}$, Fatemeh Firouzi Amoodizaj ${ }^{1}$ \& \\ Roya asI Iranifam ${ }^{1}$
}

Severe acute respiratory syndrome (SARS) is a highly contagious viral respiratory illness. This illness is spurred on by a coronavirus known as SARS-associated coronavirus (SARS-CoV). SARS was first detected in Asia in late February 2003. The genome of this virus is very similar to the SARS-CoV-2. Therefore, the study of SARS-CoV disease and the identification of effective drugs to treat this disease can be new clues for the treatment of SARS-Cov-2. This study aimed to discover novel potential drugs for SARS-CoV disease in order to treating SARS-Cov-2 disease based on a novel systems biology approach. To this end, gene co-expression network analysis was applied. First, the gene co-expression network was reconstructed for 1441 genes, and then two gene modules were discovered as significant modules. Next, a list of miRNAs and transcription factors that target gene co-expression modules' genes were gathered from the valid databases, and two sub-networks formed of transcription factors and miRNAs were established. Afterward, the list of the drugs targeting obtained sub-networks' genes was retrieved from the DGIDb database, and two drug-gene and drug-TF interaction networks were reconstructed. Finally, after conducting different network analyses, we proposed five drugs, including FLUOROURACIL, CISPLATIN, SIROLIMUS, CYCLOPHOSPHAMIDE, and METHYLDOPA, as candidate drugs for SARS-CoV-2 coronavirus treatment. Moreover, ten miRNAs including miR-193b, miR-192, miR-215, miR-34a, miR-16, miR-16, miR-92a, miR-30a, miR-7, and miR-26b were found to be significant miRNAs in treating SARS-CoV-2 coronavirus.

Coronaviruses infect both animals and humans, causing intestinal and respiratory infections ${ }^{1,2}$. Severe acute respiratory syndrome (SARS) is a coronavirus-associated respiratory disease that was originally discovered in China in February $2003^{3}$. Ten years after SARS coronavirus, Middle East respiratory syndrome (MERS) coronavirus was broke out in Middle Eastern countries ${ }^{2,4,5}$. SARS-CoV and MERS uses angiotensin-converting enzyme 2 (ACE2) and dipeptidyl peptidase 4(DPP4) as a receptor, respectively ${ }^{2}$. Additionally, in the autumn of 2019, the coronavirus SARS-CoV-2 broke out in the Chinese city of Wuhan ${ }^{4,6}$. Given that, there is a high similarity between SARS-CoV and SARS-CoV-2, the spread speed of SARS-CoV-2 is faster than SARS-CoV ${ }^{4}$. SARS-CoV-2 like SARS-CoV utilize the host cell ACE2 receptor ${ }^{4}$. ACE2 is a membrane receptor on the surface of many cell types and tissues including the lungs, heart, blood vessels, kidney, liver, and gastrointestinal ${ }^{2,4}$. SARS-CoV, SARSCoV-2, and MERS all have similar genetic characteristics, and SARS-CoV-2 is very similar to SARS-CoV ${ }^{7}$ Due to the high genetic similarity of SARS-CoV and SARS-CoV-2, the results of the study on SARS-CoV can be a clue to the treatment of SARS-CoV-2. Due to the disease's significance and high death rate, early detection and treatment are essential. The medicinal drugs used to treat coronavirus infections are only intended to be used temporarily. Besides, clinical trials on medications and vaccines that are efficient in curing diseases take quite a long time. Furthermore, handling SARS viruses in vivo is often challenging and risky. However, the knowledge obtained by sequencing their genes, proteins, or RNA is simple and easy to manage through artificial intelligence ${ }^{8}$. Additionally, miRNA-mRNA data sources have progressed considerably as prospective techniques for gaining a better understanding of potential SARS-CoV therapies, allowing network science and computational systems biology to become feasible9. According to these data, many scientists seek to identify the involved host genes and proteins in diseases to find a new therapy.

\footnotetext{
${ }^{1}$ Department of Basic Sciences, Biotechnology Research Center, Tabriz Branch, Islamic Azad University, Tabriz, Iran. ${ }^{2}$ Department of Biology, Tabriz Branch, Islamic Azad University, Tabriz, Iran. ${ }^{3}$ Department of Basic Sciences, Faculty of Veterinary Medicine, Tabriz Branch, Islamic Azad University, Tabriz, Iran. ${ }^{4}$ Department of Animal Science, Faculty of Agriculture, Tabriz Branch, Islamic Azad University, Tabriz, Iran. ${ }^{\circledR}$ email: habib_moti@ut.ac.ir
} 
Recent research has identified a set of antiviral genes, such as ISG15, IFIH1, MX1, OAS1-3, IRF7, IRF9, and STAT1 expressed by host cells, which could be used as a new therapeutic target against coronavirus due to their response to viral infection ${ }^{10-18}$. In addition to therapeutic gene targets, miRNAs can also be used to suppress the viral genome due to their ability to regulate gene expression. MiRNAs are small non-coding RNA molecules that prevent mRNAs from being translated ${ }^{19,20}$. Therefore, miRNA-based therapy could be proposed for SARS-CoV treatment ${ }^{21,22}$. On the other hand, the role of pathologic processes in miRNAs, such as inflammatory responses and viral infection, has been recently verified ${ }^{21,23,24}$.

Drug repurposing (DR) is a strategy for identifying new therapeutic uses for approved or investigational drugs $^{25,26}$. This approach is also referred to as drug reprofiling, drug re-tasking, drug repositioning, drug therapeutic and drug recyclining ${ }^{26}$. It is an efficient approach for the development or discovery of drug molecules with new therapeutic indications ${ }^{25}$. Generally, the process of drug repurposing consists of three steps ${ }^{26}: 1$. identification of a candidate molecule for a particular indication. 2. mechanistic evaluation of the drug effect in preclinical models. 3. evaluation of efficacy in phase clinical trials II. Of these three steps, step 1 is crucial, and it is here that modern approaches to hypothesising may be most useful ${ }^{26}$. These systematic approaches can be divided into experimental and computational approaches ${ }^{26}$. Some of the computational approaches are: molecular docking, signature machiing, genetic association, network mapping, retrospective clinical analysis and novel data sources ${ }^{26}$. As well as, among the experimental methods, Binding assays to identify relevant target interactions and Phenotypic screening approaches can be mentioned ${ }^{26}$.

Recently, different articles based on network approaches have been published for drug repurposing ${ }^{27}$. SAveRUNNER ${ }^{28}$ is a network-based drug repurposing algorithm, which predicts drug-disease assosiations using network-based similarity measure. This algorithm provided as a freely available R-code ${ }^{29}$. SAveRUNNER is also been used as a drug repurposing tool for amyotrophic lateral sclerosis (ALS) disease ${ }^{30}$. Pasquale and colleaqes ${ }^{31}$, examined three different network-based approaches and identified 399 repurposable drugs for COVID-19 using SAveRUNNER algorithm. Another algorithm based on artificial intelligence, network diffusion, and network proximity introduced as a drug repurposing method for SARS-CoV-2 disease ${ }^{32}$. We also recently introduced a protein-protein interaction network approach in order to propose candidate drugs to treatment of SARS-CoV-2 disease ${ }^{21}$.

Tasnimul and colleague, recently introduced a network-based method for identifying and repurposing drugs for the treatment of SARS-CoV-2 disease ${ }^{33}$. In this method, differentially expressed genes between Idiopathic pulmonary fibrosis (IPF) and SARS-CoV-2 samples were compared and finally, some IPF drugs were proposed as candidate drugs to treat SARS-CoV-2 disease. Yadi et al. ${ }^{34}$ proposed a novel network-based drug repurposing methodology based on human interactome and protein-protein interaction networks. This method quntify the interplay between the drug targets and $\mathrm{HCoV}$-host interactome in the human protein-protein interaction network. In this study, 16 potential drugs was introduced to treat SARS-CoV-2 disease. In another study, Hangyu and colleaque ${ }^{35}$ developed a machine-learning -based method to predict virus-host interactions at both organism and protein levels for SARS-Cov-2 disease. In this method, a multi-layer virus-host interaction network was constructed. $\mathrm{CoVex}^{36}$, an interactive online platform for SARS-CoV-2, introduced by Sepideh and colleaque. This platform, integrates human protein-protein interactions, virus-human protein interactions and drug-target interactions. Zhihao and colleaque ${ }^{37}$ constructed a autophagy interaction network based on competitive endogenous RNA(ceRNA) in SARS-CoV-2 infection. In this study, hsa-miR-4772-5p, hsa-miR- 192-5p, hsa-miR-652-3p, hsa-miR- 192-5p, hsa-miR-340-3p, CCR2 and TP53INP2 introduced as potential biomarkers in predicting changes in mild SARS-CoV-2 infection. In comparison to the mentioned network-based methods, in our study, the gene co-expression network is used. In addition to the gene co-expression network, regulatory interactions including miRNA-Gene, TF-Gene, and TF-miRNA have also been used in our study. Moreover, drug-gene and drug-TF interaction networks have been studied and investigated in this study. As well as, some of the genes that regulate more miRNAs, are also introduced as effective miRNAs in SARS-Cov disease. Changes in the expression of these genes can affect the expression of target miRNAs.

The current research aimed to discover the genes and miRNAs involved in SARS-CoV disease and repurpose candidate drugs for this diease in order to treating SARS-CoV-2 coronavirus based on a co-expression network analysis. In this regard, this study used a co-expression network analysis to identify potential drugs for the treatment of SARS-CoV. The methodology we used in this study is an entirely novel method based on gene module identification.

The technique first entails identifying a list of genes (human genes) expressed differentially in healthy and SARSCoV-infected samples. After obtaining differentially expressed genes between healthy and SARSCoVinfected samples, the co-expression network is reconstructed in STRING online tool ${ }^{38}$. Then, two significant gene modules are discovered from the gene co-expression network. Afterward, a list of miRNAs and transcription factor genes that have a regulatory impact on modules' genes are collected from a valid database (TRRUST v2 ${ }^{39}$ and miRWalk v $2^{40}$ ), and different network analyses are done on these biomolecules. Finally, the list of drugs that target modules' genes are gathered from the (DGIdb) ${ }^{41}$ database, and then two drug-gene interaction networks are reconstructed. The workflow diagram of this study is demonstrated in Fig. 1. As shown in this figure, the method's output is some candidate drugs for the treatment of SARS coronavirus. In this study, FLUOROURACIL, CISPLATIN, SIROLIMUS, CYCLOPHOSPHAMIDE, and METHYLDOPA are the key drugs reported for treating SARS-CoV-2 coronavirus. Moreover, $h s a-m i R-193 b$, hsa-miR-192, hsa-miR-215, hsa-miR-34a, hsa-miR-16, hsa$m i R-16, h s a-m i R-92 a, h s a-m i R-30 a, h s a-m i R-7$, and $h s a-m i R-26 b$ are candidate miRNAs, which are significant in the treatment of SARS-CoV-2 disease. 


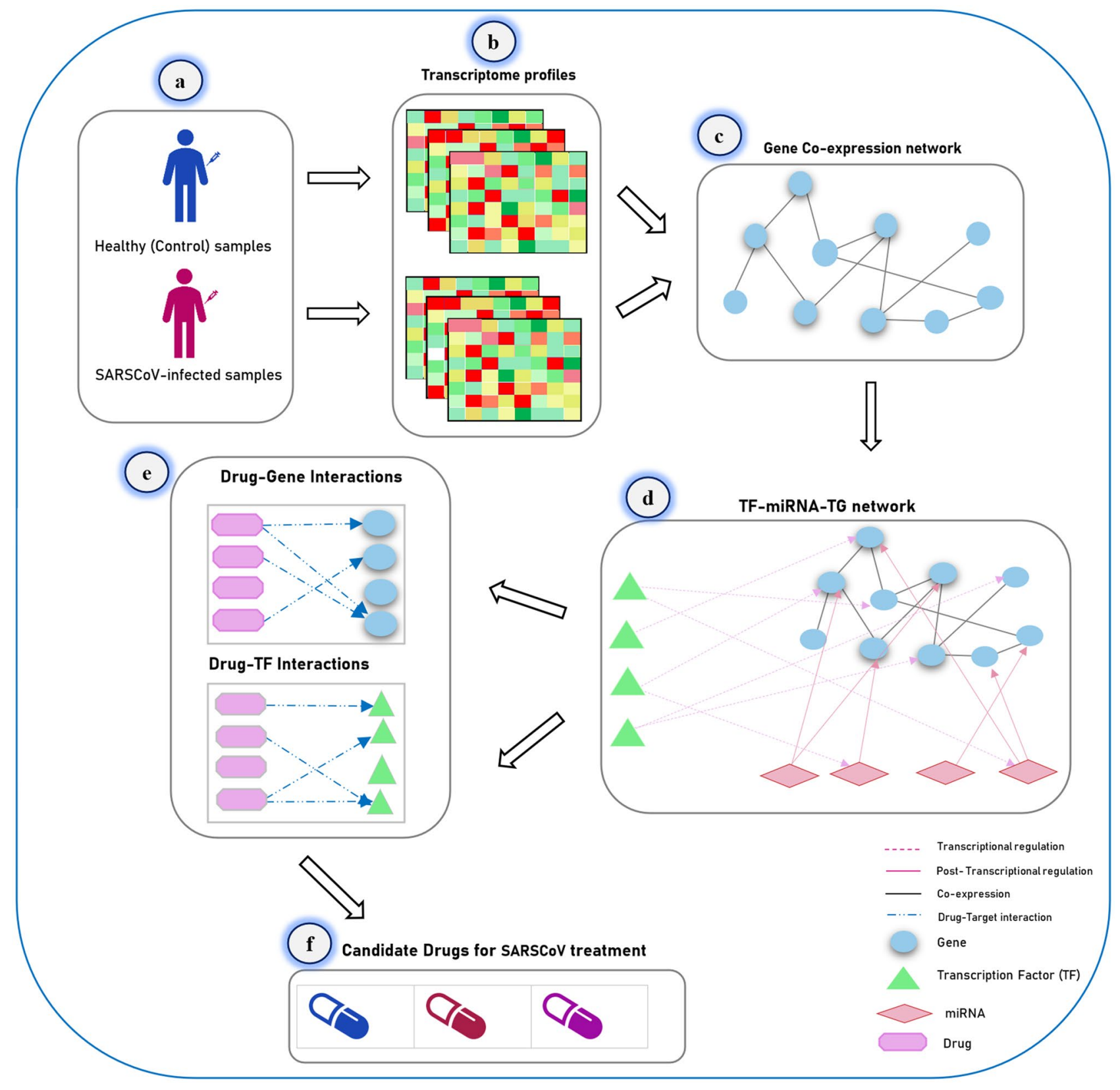

Figure 1. The overall workflow of the proposed method. In this method, a network-based approach is applied to drug repurposing for coronavirus disease treatment. (a) At first, a transcriptome profile for healthy (control) and SARS-CoV-infected samples were taken from the GEO database with the accession number GSE1739. (b) Then, after identifying differentially expressed genes in the control and disease groups, the gene co-expression network is reconstructed, and two significant gene modules are discovered from the co-expression network. (c) Next, for every gene module, the TF-miRNA-TG network is reconstructed independently. The information of TFs-miRNAs, TFs-TGs, and miRNAs-TGs regulations are taken from the TransmiR ${ }^{42}, T_{R R U S T}{ }^{39}$, and miRWalk ${ }^{40}$ databases, respectively. (d) Afterward, Drug-gene and Drug-TF networks are reconstructed for $T F-m i R N A-T G$ networks independently. (e) Finally, 19 drugs are proposed as candidate drugs for coronavirus treatment.

\section{Result}

Gene co-expression network analysis and gene modulation.. First 1441 differentially expressed genes between normal and SARS infected groups with $p$-values less than 0.05 were assumed as primary genes. Then, using this primary gene list, the gene co-expression network was reconstructed in the STRING database. In this network 1050 genes out of 1441 primary genes were disconnected. Therefore, these disconnected genes were removed from the network and a network with 391 genes was obtained. Figure 2 shows the co-expression network for these genes. Supplementary file S6 contains more information on the topological characteristics of this network. 


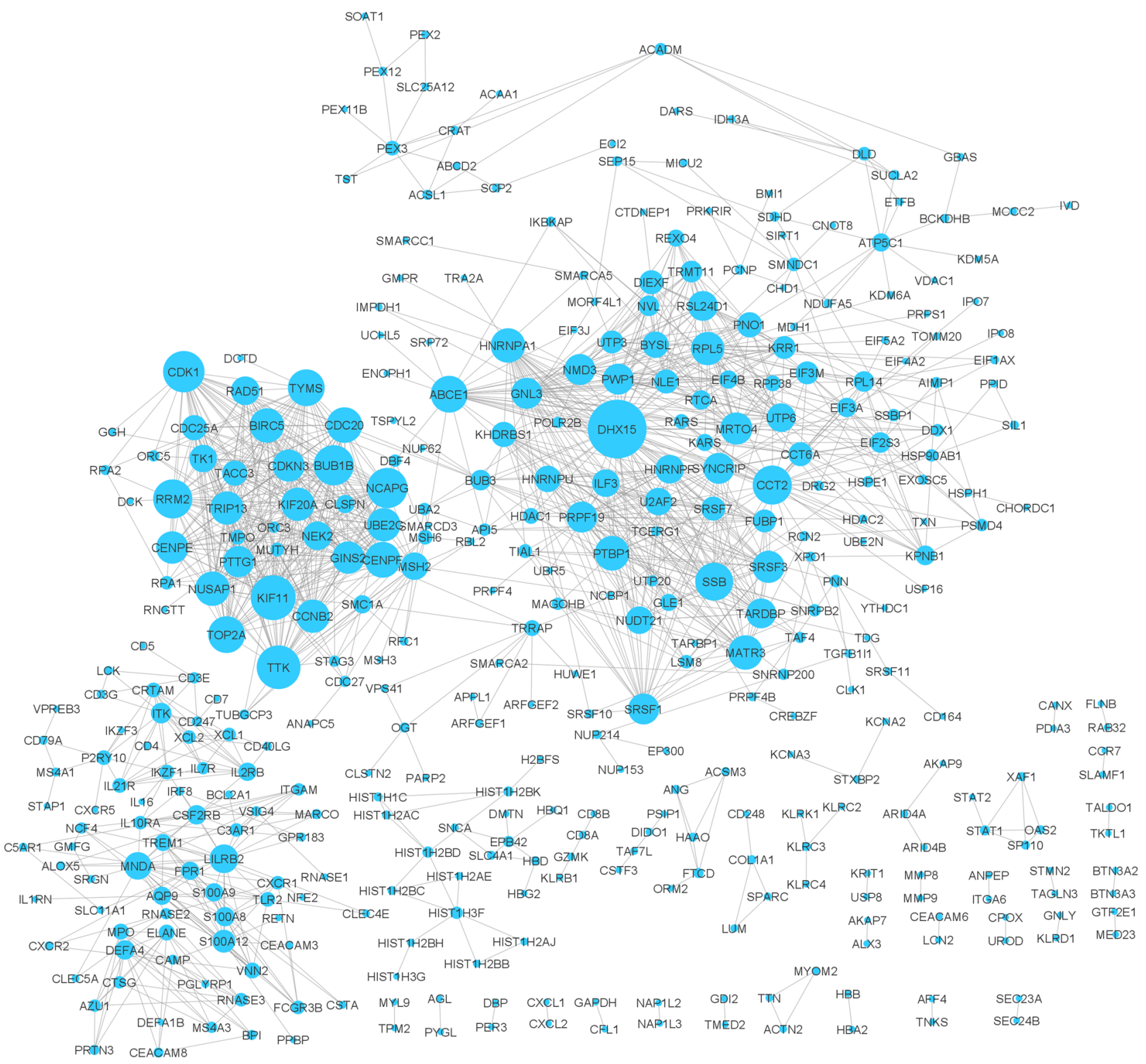

Figure 2. Gene co-expression network for the primary genes (disconnected genes were removed from the network). The size of the nodes indicates its degree. There are 391 nodes and 1273 edges in this network. DHX15 is the highest degree node in this network.

\begin{tabular}{|l|l|}
\hline Module name & Gene names \\
\hline \multirow{3}{*}{ Module A } & TOP2A (24), TTK (24), NUSAP1(24), UBE2C(24), CENPF(24), KIF20A(24), CDK1(24), CDC20(24), BIRC5(24), \\
& TRIP13(23), NCAPG(24), GINS2(23), KIF11(24), CENPE(22), BUB1B(24), CCNB2(24), CDC25A(17), TACC3(17), \\
& RAD51(20), TYMS(22), CDKN3(22), RRM2(24), TK1(19), NEK2(19), PTTG1(22) \\
\hline \multirow{3}{*}{ Module B } & SRSF1(16), SYNCRIP(14), HNRNPU(16), DHX15(29), SSB(19), MATR3(17), GNL3(13), NUDT21(16), U2AF2(17), \\
& HNRNPF(17), SRSF7(15), SRS3(17), KHDRBS1(14), FUBP1(13), PRPF19(14), BYSL(12), ILF3(17), NLE1(12), \\
& UTP6(12), RSL24D1(12), MRTO4(13), DIEXF(12), PTBP1(17), UTP3(12), PWP1(12), PNO1(12), KRR1(12), \\
& NMD3(12), HNRNPA1(17), TARDBP(17)
\end{tabular}

Table 1. Gene names of modules A and B. Numbers inside the parentheses represent the genes degree.

After analyzing the network in Cytoscape software ${ }^{43}$, generally, 391 nodes and 1273 edges were observed in the reconstructed co-expression network. After clustering the gene co-expression network, we discovered two significant modules (Module A and Module B) in the co-expression network. The list of genes for these two modules is reported in Table 1. 


\begin{tabular}{|c|c|c|c|}
\hline Module name & TF & Number of TGs & Number of target miRNAs \\
\hline TF-miRNA-TG_A & MYC & 3 & 91 \\
\hline \multirow{17}{*}{$T F-m i R N A-T G \_B$} & TP53 & 5 & 89 \\
\hline & MYC & 1 & 91 \\
\hline & NFKB1 & 1 & 67 \\
\hline & RELA & 1 & 59 \\
\hline & STAT3 & 1 & 53 \\
\hline & MYCN & 1 & 52 \\
\hline & SP1 & 4 & 38 \\
\hline & ESR1 & 1 & 30 \\
\hline & E2F3 & 1 & 20 \\
\hline & KLF4 & 1 & 18 \\
\hline & CTNNB1 & 1 & 16 \\
\hline & DNMT1 & 1 & 15 \\
\hline & NANOG & 1 & 14 \\
\hline & TP73 & 2 & 12 \\
\hline & LEF1 & 1 & 12 \\
\hline & MYB & 1 & 10 \\
\hline & FOXO3 & 1 & 9 \\
\hline
\end{tabular}

Table 2. High degree nodes of the TFs in the TF-miRNA-TG_A and B sub-networks.

Transcription factors, miRNAs, and target genes interaction network. At first two TF-miRNA$T G$ sub-networks named TF-miRNA-TG_A and TF-miRNA-TG_B were reconstructed for gene modules A and $B$, respectively. As demonstrated in previous section, two significant gene modules were considered for more analysis. To do this, the data of TFs-TGs, TFs-miRNAs, and miRNAs-TGs regulatory interactions were retrieved from the TRRUST, TransmiR, and miRWalk databases, respectively. These two sub-networks are shown in Figure S1 (See supplementary file S6). The information for TFs and TGs Interactions are activation, repression, and unknown. In addition, regulatory interactions information for TFs and TGs are: activation, activation(feedback), regulation, regulation(feedback), autoregulatory negative feedback, loop(feedback), repression, repression(feedback), auto-regulatory feedback circuit, and activation (negative regulatory loop). This information for TF-miRNA-TG_A and TF-miRNA-TG_B sub-networks (see supplementary file S7) are reported in supplementary file S2. Moreover, the topological properties for these sub-networks was reported in supplementary file S6.

In order to analyze the TFs, which regulate more Genes and miRNAs in the TF-miRNA-TG_A and TFmiRNA-TG_B networks, the high degree TF nodes were selected and reported as significant TFs. To do this, among TF nodes in the TF-miRNA-TG_A and TF-miRNA-TG_B sub-networks, the TFs with a degree of 10 and higher were selected (Table 2). The number of TGs and target miRNAs for every TF are listed in Table 2 as well.

In addition to the TFs, a list of miRNAs that regulate more genes in the TF-miRNA-TG_A and TF-miRNA$T G_{-} B$ networks were selected and reported as key miRNAs. To this end, for every TF-miRNA-TG network, five miRNAs with high degrees were assumed. These miRNAs for TF-miRNA-TG_A were hsa-miR-193b, hsa$m i R-192$, hsa-miR-215, hsa-miR-34a, and hsa-miR-16. For TF-miRNA-TG_B network, the selected miRNAs were $h s a-m i R-16, h s a-m i R-92 a, h s a-m i R-30 a, h s a-m i R-7$, and $h s a-m i R-26 b$. Among these miRNAs, hsa-miR-16 regulates more genes in both subnetworks. The list of these miRNAs, along with their degree and target genes, are reported in Table 3 .

Enrichment analysis of genes. Gene ontology was performed for the module A and B gene lists, separately. The results for module A gene list show that they significantly enriched in mitotic cell cycle process, nuclear division, and mitotic nuclear division biological processes. Moreover, the gene list of module B significantly enriched in RNA processing, mRNA metabolic process, and RNA metabolic process biological processes. Then, a pathway enrichment analysis was done for modules A and B gene lists separately. The results showed that the module A and B genes, significantly enriched in Resolution of Sister Chromatid Cohesion and mRNA SplicingMajor Pathway pathways, respectively. More details of the GO and pathway enrichment analyses are reported in supplementary file $\mathrm{S} 3$.

Enrichment analysis of miRNAs. In order to check miRNA family for the TF-miRNA-TG_A and TFmiRNA-TG_B sub-networks, at first the list of miRNAs are imported into the TAM online tool. Then, the obtained result is reported in supplementary file $\mathbf{S 4}$. As reported in this file, both sub-networks significantly enriched in the let-7 and mir-17 families.

Drug-Gene interaction network. After gathering drug-gene interactions for TF-miRNA-TG_A and TFmiRNA-TG_B genes, the drug-gene interaction network was reconstructed and is demonstrated in Fig. 3. As shown in this network, some drugs have a high degree, which means that these drugs target and regulate more 


\begin{tabular}{|l|l|l|}
\hline miRNA & Degree & Target genes \\
\hline TF-miRNA-TG_A & \multicolumn{3}{|l|}{} \\
\hline hsa-miR-193b & 13 & $\begin{array}{l}\text { RRM2, RAD51, CDC25A, CDK1, CDC20, NCAPG, KIF11, TYMS, UBE2C, TACC3, GINS2, TRIP13, } \\
\text { BUB1B }\end{array}$ \\
\hline hsa-miR-192 & 10 & RAD51, CDC25A, CDC20, CENPF, CDKN3, KIF20A, TTK, TRIP13, BUB1B, CENPE \\
\hline hsa-miR-215 & 9 & RAD51, CDC20, CENPF, CDKN3, KIF20A, TTK, TRIP13, BUB1B, CENPE \\
\hline hsa-miR-34a & 7 & RRM2, CDC25A, BIRC5, CDC20, NCAPG, KIF11, TYMS, hsa-miR-34a \\
\hline hsa-miR-16 & 7 & CDC25A, BIRC5, CDK1, CDC20, NCAPG, UBE2C, CENPF \\
\hline TF-miRNA-TG_B & \multicolumn{3}{|l}{} \\
\hline hsa-miR-16 & 8 & HNRNPF, HNRNPA1, SRSF1, NLE1, NMD3, DIEXF, UTP3, BYSL \\
\hline hsa-miR-92a & 7 & HNRNPF, PTBP1, PNO1, NLE1, NMD3, KHDRBS1, U2AF2 \\
\hline hsa-miR-30a & 6 & HNRNPA1, HRNPU, SRSF7, UTP6, PTBP1, PRPF19 \\
\hline hsa-miR-7 & 4 & HNRNPU, MATR3, SRSF1, hsa-miR-7, ILF3 \\
\hline hsa-miR-26b & 4 & MATR3, SYNCRIP, NMD3, GNL3 \\
\hline
\end{tabular}

Table 3. High degree nodes of the miRNAs in the TF-miRNA-TG_A and $B$ sub-networks.

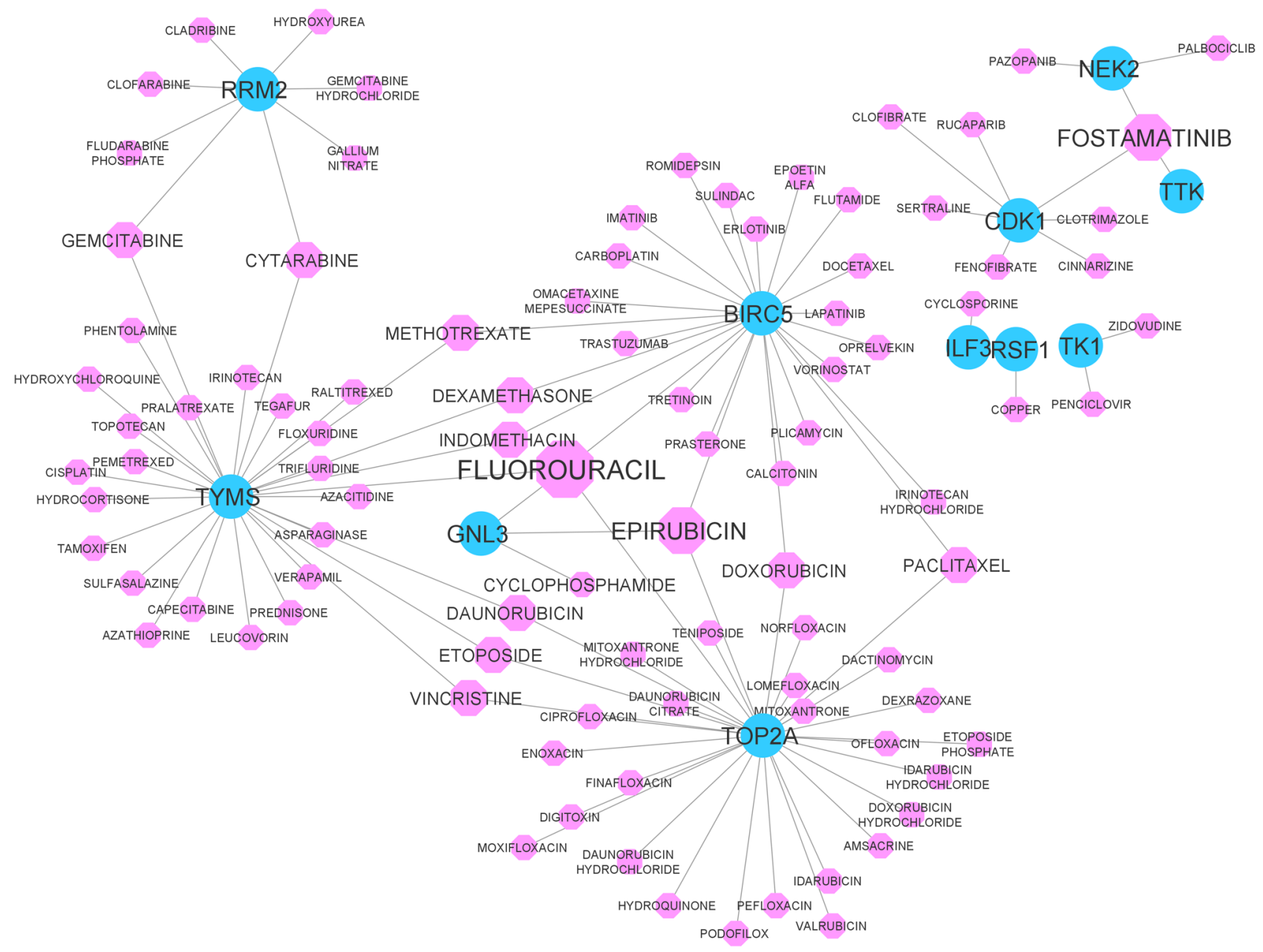

Figure 3. The drug-gene interaction network for TF-miRNA-TG_A and TF-miRNA-TG_B sub-networks. Blue circles show TGs, and pink octagons show drugs. The size of the octagon nodes indicates its degree. The high degree drug is FLUOROURACIL with 4 target genes.

genes. Therefore, high-degree drugs were selected and reported as significant, as they regulate more genes in module_A and module_B. In this regard, the drugs with a degree of 3 or higher were selected and are reported in Table 4. As reported, FLUOROURACIL, EPIRUBICIN, and FOSTAMATINIB are effective drugs and targeted 4,3 , and 3 genes, respectively. 


\begin{tabular}{|l|l|l|}
\hline Drug & Degree & Target genes \\
\hline FLUOROURACIL & 4 & BIRC5, GNL3, TYMS, TOP2A \\
\hline EPIRUBICIN & 3 & BIRC5, GNL3, TOP2A \\
\hline FOSTAMATINIB & 3 & NEK2, CDK1, TTK \\
\hline
\end{tabular}

Table 4. High degree drugs in the drug-gene network. Of all the drugs, only those with degree 3 or higher have been reported.

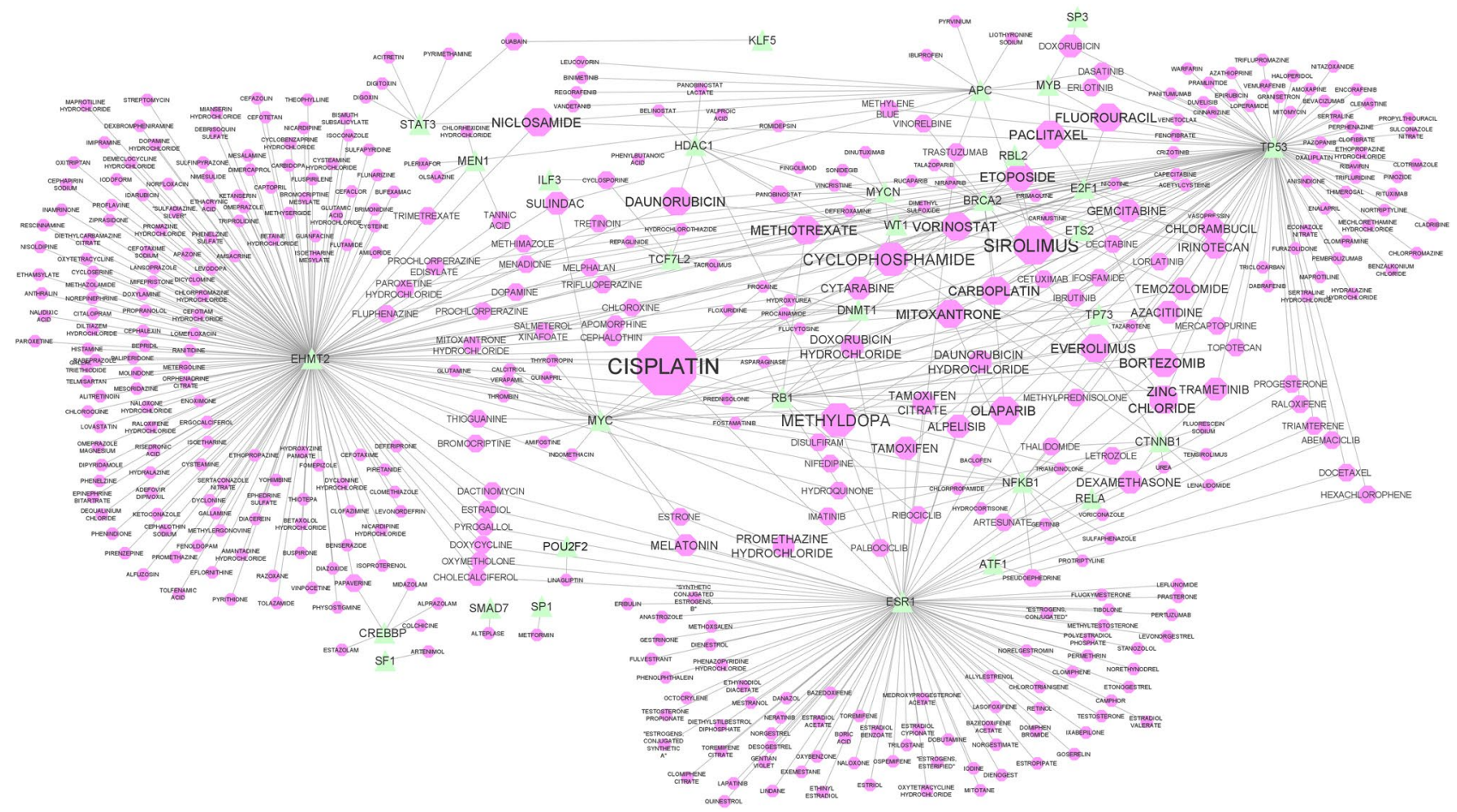

Figure 4. Drug-TF interaction network for TF-miRNA-TG_A and TF-miRNA-TG_B sub-networks. Green triangle shapes show genes, and pink octagon shapes show drugs. The size of the octagon nodes indicates its degree. The high degree drug is CISPLATIN with 11 target genes.

In addition to the Drug-gene interaction network, the drug-TF network was reconstructed to discover the drugs that target the transcription factors of the TF-miRNA-TG_A and TF-miRNA-TG_B sub-networks. This network is shown in Fig. 4. In this network, the drugs with a degree of 4 and higher were selected and reported as significant drugs. More details of the effective drugs are reported in Table 5. As the table shows, CISPLATIN, SIROLIMUS, and CYCLOPHOSPHAMIDE drugs targeted 11,6, and 5 TFS, respectively, and the others targeted 4 TFs. The complete information of Drug-Gene and Drug-TF interactions are provided in supplementary file S5. Additionally, supplemental file S6 has detailed information on the network topological characteristics of the Drug-Gene and Drug-TF networks.

Recently, the potential effect of 6710 drugs as SARS-CoV-2 inhibitors is tested in vitro and in vivo ${ }^{44}$. The results of this report show that some of the drugs proposed in this article (see Tables 4 and 5) have an inhibitory effect on SARS-CoV-2. According to this report, OLAPARIB, NICLOSAMID and METHOTREXATE have a very weak, weak, and strong effects on SARS-CoV-2, respectively. As well as, DAUNORUBICIN and BORTEZOMIB have a cytotoxic effect on this disease.

GSEA and candidate drugs validation. To evaluate the proposed drugs, the Connectivity Map(CMAP) analysis was utilized. To do this, from the Drug-Gene and Drug-TF networks, the drugs with a degree of 4 or above were selected and imported to Enrichr CMAP database. Then, the impact of these drugs on target genes was evaluated. Enrichr CMAP database contains CMAP-up and CMAP-down datasets. In Tables 3 and 4, from among the 19 repurposed drugs for SARS-CoV disease, only nine drugs, including SIROLIMUS, METHYLDOPA, VORINOSTAT, PACLITAXEL, DAUNORUBICIN, METHOTREXATE, NICLOSAMIDE, and ETOPO$S I D E$, were validated by CMAP analysis. Table 6 shows the validated drugs together with the corresponding unregulated or downregulated target genes. 


\begin{tabular}{|l|l|l|}
\hline Drug & Degree & Target TFs \\
\hline CISPLATIN & 11 & BRCA2, DNMT1, E2F1, EHMT2, ESR1, MYC, MYCN, RB1, TP53, TP73 \\
\hline SIROLIMUS & 6 & APC, RB1, RBL2, TCF7L2, TP53, WT1 \\
\hline CYCLOPHOSPHAMIDE & 5 & BRCA2, CTNNB1, EHMT2, MYCN, TP53 \\
\hline METHYLDOPA & 5 & EHMT2, ESR1, HDAC1, TP53, TP73 \\
\hline VORINOSTAT & 4 & HDAC1, MYC, RB1, TP53 \\
\hline OLAPARIB & 4 & BRCA2, ESR1, MYC, TP53 \\
\hline MITOXANTRONE & 4 & DNMT1, EHMT2, NFKB1, TP53 \\
\hline FLUOROURACIL & 4 & APC, E2F1, MYB, TP53 \\
\hline EVEROLIMUS & 4 & BRCA2, CTNNB1, ESR1, RB1 \\
\hline PACLITAXEL & 4 & BRCA2, E2F1, MYB, TP53 \\
\hline DAUNORUBICIN & 4 & EHMT2, HDAC1, TP53, WT1 \\
\hline ZINC CHLORIDE & 4 & ESR1, HDAC1, TP53, TP73 \\
\hline METHOTREXATE & 4 & E2F1, EHMT2, RB1, TP53 \\
\hline CARBOPLATIN & 4 & BRCA2, ETS2, TP53, TP73 \\
\hline BORTEZOMIB & 4 & E2F1, NFKB1, RB1, TP53 \\
\hline NICLOSAMIDE & 4 & APC, EHMT2, STAT3, TP53 \\
\hline ETOPOSIDE & 4 & BRCA2, E2F1, MYCN, TP53 \\
\hline
\end{tabular}

Table 5. High degree drugs in the Drug-TF network. Of all the drugs, only those with degree 4 or higher have been reported.

\begin{tabular}{|c|c|}
\hline Drug name & Gene names ( $\downarrow$ :Downregulated and $\uparrow:$ upregulated) \\
\hline SIROLIMUS & TYMS $(\uparrow)$, NLE1 $(\uparrow)$, CDC25A $(\uparrow), \operatorname{BYSL}(\downarrow)$, UTP3 $(\downarrow)$, GNL3 $(\downarrow)$, MRTO4 $(\downarrow)$, UTP6 $(\downarrow)$, KRR1 $(\downarrow)$, CDC25A $(\downarrow)$ \\
\hline METHYLDOPA & NMD3 ( $)$ \\
\hline VORINOSTAT & $\mathrm{CCNB} 2(\downarrow)$ \\
\hline PACLITAXEL & KHDRBS1 ( $\uparrow$ \\
\hline DAUNORUBICIN & BUB1B $(\downarrow)$, CENPE $(\downarrow)$, NMD3 $(\downarrow)$ \\
\hline METHOTREXATE & $\begin{array}{l}\text { UBE2C }(\downarrow), \text { CDC20 }(\downarrow), \text { BUB1B }(\downarrow), \text { KIF20A }(\downarrow) \text {, PTTG1 }(\downarrow), \text { CDKN3 }(\downarrow) \text {, KIF11 }(\downarrow) \text {, TACC3 }(\downarrow) \text {, TOP2A }(\downarrow), \text { TTK } \\
(\downarrow), \text { CENPF }(\downarrow), \text { CCNB2 }(\downarrow)\end{array}$ \\
\hline NICLOSAMIDE & HNRNPA1 ( $\uparrow$ ) GINS2 ( $\downarrow)$, BYSL $(\downarrow)$, RRM2 $(\downarrow)$ \\
\hline ETOPOSIDE & $\begin{array}{l}\text { UBE2C }(\downarrow), \text { CDC20 }(\downarrow), \text { KIF20A }(\downarrow), \text { NCAPG }(\downarrow) \text {, PTTG1 }(\downarrow) \text {, PNO1 }(\downarrow) \text {, NEK2 }(\downarrow), \text { CENPF }(\downarrow) \text {, TOP2A }(\downarrow), \text { KIF1 } 1 \\
(\downarrow), \text { CDKN3 }(\downarrow), \text { TACC3 }(\downarrow) \text {, TTK }(\downarrow)\end{array}$ \\
\hline
\end{tabular}

Table 6. The validated candidate drugs by CMAP analysis.

\section{Discussion}

In this article, a network-based approach was applied to discover therapeutic drugs for SARS-CoV-2 disease. Due to the high genetic similarity of SARS-CoV and SARS-CoV-2, the results of the study on SARS-CoV can be a clue to the treatment of SARS-CoV-2. To this end, at first, differentially expressed significant genes $(P$-value $<0.05)$ in healthy and SARS-CoV infected samples were selected, then the gene co-expression network was reconstructed in STRING database for the filtered genes.

After reconstructing the gene co-expression network, we discovered two significant gene modules using ClusterViz plugin in Cytoscape software. These two obtained gene co-expression modules (Module A and Module $B$ ) contained 25 and 30 genes, respectively. In the next step, a list of TFs and miRNAs which regulate these module's genes were gathered from the TRRUST and miRWalk2.0 databases, respectively. Moreover, the regulation information of the TFs and miRNAs were obtained from the TransmiR database. After collecting the TFs, miRNAs, and TGs regulation information, the two sub-networks of TF-miRNA-TG_A and TF-miRNA-TG_B were reconstructed. TF-miRNA-TG_A contains 347 miRNAs, 25 TGs, and 57 TFs, and TF-miRNA-TG_B contains 116 miRNAs, 25 TGs, and 4 TFs.

To analyze the biological processes and pathways in which Module A and Module B are involved, Gene Ontology (GO) and pathway enrichment analyses were done using a DAVID tool. The GO enrichment analysis showed that the genes in Module A significantly enriched in mitotic cell cycle process, nuclear division, and mitotic nuclear division biological processes. In Module B, the genes significantly enriched in RNA processing, mRNA metabolic process, and RNA metabolic process terms. The Reactome pathway enrichment analysis showed that modules A and $B$ enriched in Resolution of Sister Chromatid Cohesion and mRNA Splicing-Major Pathway terms, respectively (see supplementary file S3). To analyze the TF-miRNA-TG_A and TF-miRNA-TG_B sub-network's miRNAs, the TAM online tool was utilized. Using this tool, we identified miRNAs families for these sub-networks. 
The results showed that the miRNAs of both sub-networks significantly enriched in let-7 and mir-17 families. Some other significant miRNAs families are reported in the supplementary file S4.

Given that the transcription factors have considerable roles in the gene expression process, the TFs of both TF-miRNA-TG_A and TF-miRNA-TG_B sub-networks were studied and evaluated. To this end, from any TFmiRNA-TG sub-networks, the TFs with a degree of 10 and above were selected and reported. The high-degree TFs were MYC, TP53, NFKB1, RELA, STAT3, MYCN, SP1, ESR1, E2F3, KLF4, CTNNB1, DNMT1, NANOG, TP73, LEF1, MYB, and FOXO3. These 17 transcription factor genes can be evaluated in further studies on SARSCoV-2 coronavirus disease.

Hub miRNAs in TF-miRNA-TG_A and TF-miRNA-TG_B sub-networks are essential and crucial, as they regulate more genes of the subnetworks at a post-translational regulation level and can impact biological processes. Therefore, for each TF-miRNA-TG subnetwork, five high degree miRNAs were selected and reported as significant miRNAs. Based on the findings, hsa-miR-193b, hsa-miR-192, hsa-miR-215, hsa-miR-34a, and hsamiR-16 were significant miRNAs for TF-miRNA-TG_A subnetwork, and hsa-miR-16, hsa-miR-92a, hsa-miR-30a, $h s a-m i R-7$, and $h s a-m i R-26 b$ were found to be significant miRNAs for TF-miRNA-TG_B subnetwork as well. Among these miRNAs, $h s a-m i R-16$ regulates more genes in both subnetworks. According to the literature, some of these miRNAs have been studied in SARS coronavirus.

Kyung Hee Choi et al. ${ }^{45}$ found that has-miR-193 is a dual-strand tumor suppressor and a novel therapeutic target for lung cancer. In another study, Huajun $\mathrm{Hu}$ et al. ${ }^{46}$ reported that this miRNA is a tumor suppressor in Non-small cell lung cancer (NSCLC). Liang Sun et al. ${ }^{47}$ concluded that hsa-miR-193b regulates the RAB22A oncogene, inhibits breast cancer growth, and may have significant implications for cancer therapy. In addition, this miRNA regulates breast cancer cell migration and vasculogenic mimicry by DDAH $1^{48}$. Moreover, it could function as a tumor-suppressive miRNA in breast cancer ${ }^{49}$ and inhibits breast cancer metastasis ${ }^{50}$.

Martyna Filipska et al. ${ }^{51}$ found that hsa-miR-192-5p has a functional role in squamous cell lung cancer cells. In Peng Zou et al. ${ }^{52}$ reported that this miRNA suppresses the progression of lung cancer bone metastasis by targeting TRIM44. Moreover, this miRNA has been introduced as a prognostic marker for NSCLC participants ${ }^{53}$. Importantly, hsa-miR-192 induces Cisplatin-resistance, inhibits cell apoptosis in lung cancer ${ }^{54}$ and the proliferation, migration, and invasion of osteosarcoma cells, and promotes apoptosis ${ }^{55}$. Xiaopan et al ${ }^{56}$ reported that $h s a-m i R-215$ suppresses proliferation and migration of non-small cell lung cancer cells(NSCLC). This miRNA is downregulated in NSCLC tissues and may play a key role in the development of NSCLC. The lower expression of has-miR-215 in NSCLC is negatively associated with lymphatic metastasis and TNM staging ${ }^{57}$. This miRNA targets ZEB2 in human non-small cell lung cancer and functions as a tumor suppressor ${ }^{58}$. Ariana Centa et al. ${ }^{59}$ expressed that has-miR-34a-5p is identified as the regulator of mRNA targets involved in endothelial, inflammatory signaling pathways, and viral diseases. Furthermore, in the present study, the expression of this miRNA was significantly down-regulated in the COVID-19 patients compared to the Controls. Also, Martin Hart et al. ${ }^{60}$, in their systems biology, analysis identified $m i R-34 a$ as strongly associated with pathogenesis. In another study, Rieko Aida et al. ${ }^{61}$ reported that apigenin might induce apoptosis by down-regulating SNAI1 through $m i R$ $34 a-5 p$ up-regulation in A549 cells. Woo Ryung Kim et al. ${ }^{62}$ reported that $h s a-m i R-16-5 p$ is commonly bound to SARS-CoV, MERS-CoV, and SARS-CoV-2. In Zofia Wicik et al. ${ }^{63}$ showed that this miRNA could regulate ACE2 networks. Moreover, this miRNA can link the pathogenesis of HIV-1 and malaria ${ }^{64-66}$. Similarly, Jianghong Wei et al. ${ }^{67}$ found that overexpression of miR-16 inhibited the growth and metastasis of the DMS-53 lung cancer cells. Alireza Paniri et al. ${ }^{68}$ reported that $h s a-m i R-26 b-5 p$ strongly targets ACE2 and have an important effect on SARS coronavirus. Like has-miR-16-5p, has-mir-26b-5p can regulate ACE2 networks as well ${ }^{63}$. Moreover, this paper reported that has-miR-26b-5p may plays a significant role in the pathogenesis of HF in COVID-19 patients. The effect of this miRNA in SARS coronavirus was studied by Laura Teodori and her colleagues ${ }^{69}$. Moreover, Yang Gao et al. ${ }^{70}$ reported that this miRNA plays an important role in tumor suppression in lung cancer. According to M Xia et al., this miRNA could suppress lung cancer cells' proliferation, migration, and invasion. Min Jiang et al. ${ }^{71}$ reported that has-miR-92a family could be ideal biomarkers for cancer diagnosis and prognosis. Also, our study revealed that the expression of has-mir-92a was upregulated in lung squamous cell carcinoma (LUSC). Besides, this miRNA could promote growth, metastasis, and chemoresistance in NSCLC cells ${ }^{72}$. This miRNA was thus introduced as a plasma biomarker for small cell lung cancer ${ }^{73}$. Jianhua Gong et al. ${ }^{74}$ revealed that the hasmiR-92a up-regulation could significantly induce proliferation and inhibit apoptosis of lung cancer cells. Jianjie Zhu et al. ${ }^{75}$ revealed that the upregulation of has-mir-30-5p in lung cancer cell lines inhibited cell proliferation in vitro and in vivo. This miRNA suppresses lung cancer progression by targeting SIRT1 ${ }^{76}$. Also, the lack of its expression promotes the growth of lung cancer cells by targeting MEF2D. Moreover, Xiaowei Quan et al. ${ }^{77}$ and Ruixue Tang et al. ${ }^{78}$ revealed that miR-30a-5p expression is downregulated in NSCLC. In addition, the increase in miR-30a-5p level could enhance Bax protein level and decrease Bcl-2 protein level ${ }^{77}$. In the field of pharmaceutical research, Xiaojie Xu et al..$^{79}$ reported that miR-30a-5p enhances paclitaxel sensitivity in non-small cell lung cancer through targeting BCL-2 expression. Haiping Xiao et al. ${ }^{80}$ believed that has-miR-7-5p suppresses tumor metastasis of NSCLC by targeting NOVA2. Plus, Kenneth Lundstrom ${ }^{81}$ revealed that Rotavirus (RV) miR-7 can inhibit rotavirus replication by targeting the RV nonstructural protein 5. In another study, it was found that has-miR-7 could repress fibrogenesis of lung fibroblasts induced by TGF- $\beta 1^{82}$. In addition, Xiaofei Zhang et al. ${ }^{83}$ reported that the overexpressed CDRlas functions as an oncogene to promote the tumor progression via miR-7 in non-small-cell lung cancer.

From the perspective of pharmacological studies, our finding shows that 470 drugs target $\mathrm{TF}$ and non-TF genes in both TF-miRNA-TG_A and TF-miRNA-TG_B subnetworks. Of 470 drugs, 62 drugs target both TF and non-TF genes, 95 drugs target non-TF genes, and 436 drugs target TF genes. From among the 470 obtained drugs, the drugs which target more genes were selected and discussed. In the drug-gene network, the drugs with a degree of 3 or above were selected and reported as potential and effective drugs for treating patients infected with SARS coronavirus. These drugs, including FLUOROURACIL, EPIRUBICIN, and FOSTAMATINIB, target 
at least three genes in the drug-gene network. Also, the drugs with a degree of 4 or above were selected and reported for the drug-TF network. These high-degree drugs were CISPLATIN, SIROLIMUS, CYCLOPHOSPHAMIDE, METHYLDOPA, VORINOSTAT, OLAPARIB, MITOXANTRONE, FLUOROURACIL, EVEROLIMUS, PACLITAXEL, DAUNORUBICIN, ZINC CHLORIDE, METHOTREXATE, CARBOPLATIN, BORTEZOMIB, NICLOSAMIDE, and ETOPOSIDE. Of the reported drugs, CISPLATIN targets 11 Transcription factor genes and may have a crucial impact on SARS coronavirus disease. We found that some of these drugs have been studied in SARS-CoV and SARS-CoV-2 coronaviruses, and others can be assumed as candidate drugs for SARS-CoV-2 coronavirus disease therapeutic.

Shamim I. Ahmad, in his recent study, revealed that FLUOROURACIL, in combination with deoxyribose and deoxyribonucleosides, can be a therapeutic option for SARS coronavirus ${ }^{84}$. EPIRUBICIN, VAPREOTIDA, and SAQUINAVIR have been proposed as key drugs in SARS coronavirus treatment ${ }^{85}$. Also, Strich et al. ${ }^{86}$ introduced the FOSTAMATINIB as a potential therapeutic for COVID-19. Moreover, FOSTAMATINIB has the potential to treat serious outcomes of coronavirus COVID-19, including acute lung injury (ALI) and acute respiratory distress syndrome (ARDS) ${ }^{87,88}$. In addition, several studies evaluated and showed the impact of FOSTAMATINIB on SARS coronavirus ${ }^{89-92}$. The mTOR signaling plays a crucial role in MERS-CoV infection ${ }^{93}$. In this regard, Yadi Zhou et al. ${ }^{34}$ observed that the SIROLIMUS is an inhibitor of mTOR with both antifungal and antineoplastic properties. In addition, this drug has been presented as a viral protein expression blocker ${ }^{94}$. Swaroop Revannasiddaiaha et al. ${ }^{95}$ showed that CYCLOPHOSPHAMIDE had a potential role in mitigation of acute respiratory distress syndrome among patients with SARS-CoV-2. Moreover, Brocato et al. ${ }^{96}$, Othenin-Girard et al..$^{97}$, Corso et al..$^{98}$, Schaecher et al. ${ }^{99}$, and Revannasiddaiah et al. ${ }^{95}$ evaluated and showed the impact of CYCLOPHOSPHAMIDE on SARS coronavirus disease with different approaches.

Al-Rashedi et al. ${ }^{100}$ noted that the OLAPARIB is a potential drug for treating patients infected with SARSCOV-2. MITOXANTRONE has also been introduced as potential inhibitors of SARS-CoV-2 Mpro101 . Safavi et al. ${ }^{102}$ showed that the METHOTREXATE silence the immune activation in patients with COVID-19. Also, Sujoy Khan et al. ${ }^{103}$ proposed this drug as a potential drug for treating patients infected with COVID-19. Additionally, this drug has a protective effect on SARS-CoV-2 infection via downregulating ACE2 ${ }^{104}$. In this study, another drug that we have reported as a potential drug for SARS coronavirus was NICLOSAMIDE. This drug has previously been reported as an antiviral agent against COVID-19 ${ }^{105,106}$. Other studies have reported the potential of this drug in treating patients infected with COVID-19107,108. Different studies have been undertaken on the effect of ETOPOSIDE on SARS coronavirus disease ${ }^{109-112}$. In our previous research based on protein-protein-network analysis, we proposed PACLITAXEL, CARBOPLATIN, BORTEZOMIB, VORINOSTAT, and DAUNORUBICIN as potential drugs for SARS-CoV-2 coronavirus treatment ${ }^{21}$. In this study, PACLITAXEL was introduced as the most potent therapeutic candidate drug. In previous research, rare studies examined the effect of this drug on SARS-CoV-2 disease.

In conclusion, based on our results, these 19 drugs can be assumed as candidate therapeutic drugs for SARSCoV-2 coronavirus. Moreover, along with some other drugs, nine miRNAs were proposed as candidate miRNAs, which may play an important role in treating SARS-CoV-2 disease. These candidate miRNAs include hsa-miR-193b, hsa-miR-192, hsa-miR-215, hsa-miR-34a,hsa-miR-16, hsa-miR-92a, hsa-miR-30a, hsa-miR-7, and hsa-miR-26b.

\section{Conclusion}

In this study, focusing on the gene expression profile of SARS-CoV samples, an attempt was made to identify effective drugs for the treatment of this disease with a gene co-expression network-based approach. Given that the genomes of SARS-CoV and SARS-CoV-2 are very similar, it is expected that the drugs introduced to treat SARS-CoV coronavirus would also be effective in treating SARS-CoV-2 disease. Current research aimed to discover novel potential drugs for SARS-CoV disease in order to treating SARS-CoV-2 coronavirus based on a co-expression network analysis. To this end, at first, significant DEGs in normal and SARS-CoV infected samples were selected and then the gene co-expression network was reconstructed and two gene modules were discovered as significant modules. Then, two significant gene modules were discovered from the reconstructed co-expression network. Next, for the obtained modules, two sub-networks named TF-miRNA-TG_A and TFmiRNA-TG_B were drawn. Afterward, the list of the drugs targeting TF-miRNA-TG_A and TF-miRNA-TG_B sub-networks' genes was extracted, and two drug-gene and drug-TF interaction networks were drawn. Eventually, five drugs including FLUOROURACIL, CISPLATIN, SIROLIMUS, CYCLOPHOSPHAMIDE, and METHYLDOPA are proposed as poteintial drugs for SARS-CoV-2 coronavirus treatment. As well as, ten miRNAs including $m i R-193 b, m i R-192, m i R-215, m i R-34 a, m i R-16, m i R-16, m i R-92 a, m i R-30 a, m i R-7$, and $m i R-26 b$ were found to be significant miRNAs in treating SARS-CoV-2 coronavirus.

\section{Methods}

Dataset and preprocessing. The gene expression data used in this work were downloaded from the NCBI Gene Expression Omnibus (GEO) database with the accession number GSE1739. This data contains gene expression profiles of normal and Severe Acute Respiratory Syndrome (SARS) infected patients' blood samples. To assign probes to gene IDs, the annotation file published by Affymetrix was used.

In this article, a network-based approach was applied to discover therapeutic drugs for SARS-CoV-2 disease. To this end, at first, differentially expressed significant genes ( $p \_$value $\left.<0.05\right)$ in healthy and SARS-CoV infected samples were selected, and then the gene co-expression network was reconstructed in STRING database for the filtered genes. 
Network reconstruction and module extraction. At first, differentially expressed genes were extracted for the normal and SARS infected groups. In order to calculate the differentially expressed genes, adjusted $\mathrm{p}_{-}$ value was calculated using Benjamini \& Hochberg false discovery rate method. Then, 1441 genes with $p$-values less than 0.05 were assumed as primary genes, which were then used in network reconstruction. The list of mentioned primary genes is reported in Supplementary file S1. Afterward, the gene co-expression network is reconstructed by primary genes in STRING database. In this web tool, the minimum required interaction score parameter is adjusted to 0.04 .

In order to analyze the reconstructed co-expression network, the Cytoscape software ${ }^{43}$ version 3.8.2 was used. ClusterViz ${ }^{113}$ plugin was used to identify gene modules (highly interconnected regions) in the co-expression network. ClusterViz is a Cytoscpae plugin, which discovers modules in a biological network using various clustering algorithms. This plugin contains three commonly used clustering algorithms, including FAG-EC, EAGLE, and MCODE.

In this research, we have applied all of the algorithms and the results did not have significant difference, so we have decided to select one of them. Therefore, the MCODE (Molecular Complex Detection) algorithm was used to find the gene co-expression modules. MCODE is a graph theoretic clustering algorithm for discovering strongly connected regions in a given network ${ }^{113}$. This algorithm selects the seed nodes and expand them based on the density of the cluster and density of the local neighborhood ${ }^{113}$. The MCODE algorithm was performed with the following parameters: Degree threshold $=2$, NodeScore Threshold $=0.2, \mathrm{~K}$-Core Threshold $=2$, and Maxdepth $=100$.

Transcription factors, miRNAs, and target genes interaction network. To investigate the effect of Transcription Factors (TF) and microRNAs (miRNA) on target genes (TG), the TF-miRNA-TG sub-networks were reconstructed for gene modules.

Transcription factors (TF) are proteins that regulate the rate of transcription of genetic information from DNA to messenger RNA ${ }^{114}$. miRNAs are small non-coding RNAs that function in RNA silencing and post-transcriptional gene regulation ${ }^{23,115}$. Both TFs and miRNAs regulate gene expression ${ }^{116}$. To get regulatory interactions information of TFs-TGs, the TRRUST online database was utilized. This database contains 8,444 and 6,552 TF-target regulatory relationships of 800 human TFs and 828 mouse TFs, respectively. In addition to TFs-TGs regulatory information, TFs-miRNAs regulatory interactions are essential. This information is obtained from the TransmiR V2 ${ }^{42}$ database. TransmiR v2.0 incorporates 3,730 TF-miRNA regulatory interactions, covering 623 TFs and 785 miRNAs for 19 organisms. In this study, the information of miRNAs-TGs regulatory interactions was retrieved from the miRWalk 2.0 database. This database contains both validated and predicted interactions. In this study, only experimentally validated miRNAs-TGs interactions were considered.

In order to analyze the reconstructed co-expression network, the Cytoscape software was used. After analyzing the network, generally, 391 nodes and 1273 edges were observed in the reconstructed co-expression network. ClusterViz ${ }^{113}$ plugin was used to identify gene modules (highly interconnected regions) in the co-expression network. ClusterViz is a Cytoscpae plugin, which discovers modules in a biological network using various clustering algorithms. This plugin contains three commonly used clustering algorithms, including FAG-EC, EAGLE, and MCODE. The MCODE (Molecular Complex Detection) algorithm was used to find the gene co-expression modules in this study. The MCODE algorithm was performed with the following parameters: Degree threshold $=2$, NodeScore Threshold =0.2, K-Core Threshold =2, and Maxdepth $=100$. After clustering the gene co-expression network, we discovered two significant modules (Module A and Module B) in the co-expression network.

Enrichment analysis. The Database for Annotation, Visualization, and Integrated Discovery (DAVID ) v6. $8^{117,118}$ was used for the enrichment analysis of the genes. The gene ontology and pathway enrichment analysis were done for obtained gene modules.

To identify miRNAs family, the TAM online tool ${ }^{119}$ was applied. To do this, all the miRNAs in TF-miRNA-TG sub-networks were imported to the TAM tool separately, and then significant miRNA families were identified.

Drug-Gene interaction network. To identify the drugs that target TF-miRNA-TG_A and TF-miRNA$T G_{-} B$ TF and non-TF genes, the Drug Gene Interaction Database (DGIdb) ${ }^{41}$ was used. This database retrieves drug-gene interaction information from 24 other related databases. In this study, to identify drug-genes interaction, only approved drugs were used.

GSEA and candidate drugs validation. In order to evaluate the proposed drugs for SARS-CoV-2 disease, the GSEA was performed by querying the Enrichr database ${ }^{120}$. To this end, the Enrichr database was utilized to perform the Connectivity Map(CMAP) analysis ${ }^{121}$.

Received: 14 August 2021; Accepted: 28 October 2021

Published online: 08 November 2021

\section{References}

1. Masters, P. \& Perlman, S. (Lippincott Williams \& Wilkins, 2013).

2. Cui, J., Li, F. \& Shi, Z.-L. Origin and evolution of pathogenic coronaviruses. Nat. Rev. Microbiol. 17, 181-192 (2019).

3. McBride, R. \& Fielding, B. C. The role of severe acute respiratory syndrome (SARS)-coronavirus accessory proteins in virus pathogenesis. Viruses 4, 2902-2923 (2012). 
4. Rabaan, A. A. et al. SARS-CoV-2, SARS-CoV, and MERS-COV: a comparative overview. Infez Med 28, 174-184 (2020).

5. Zaki, A. M., Van Boheemen, S., Bestebroer, T. M., Osterhaus, A. D. \& Fouchier, R. A. Isolation of a novel coronavirus from a man with pneumonia in Saudi Arabia. N. Engl. J. Med. 367, 1814-1820 (2012).

6. El-Hachem, N. et al. Integrative transcriptome analyses empower the anti-COVID-19 drug arsenal. Iscience 23, 101697 (2020).

7. Zhu, N. et al. A novel coronavirus from patients with pneumonia in China. New England J. Med. 2, 1056 (2020).

8. MotieGhader, H., Gharaghani, S., Masoudi-Sobhanzadeh, Y. \& Masoudi-Nejad, A. Sequential and mixed genetic algorithm and learning automata (SGALA, MGALA) for feature selection in QSAR. Iran. J. Pharm. Res. IJPR 16, 533 (2017).

9. Wu, Q., Coumoul, X., Grandjean, P., Barouki, R. \& Audouze, K. Endocrine disrupting chemicals and COVID-19 relationships: A computational systems biology approach. Environ. Int. 12, 106232 (2020).

10. Wei, J. et al. Genome-wide CRISPR screen reveals host genes that regulate SARS-CoV-2 infection. Biorxiv (2020).

11. Chakraborty, C. et al. Consider TLR5 for new therapeutic development against COVID-19. J. Med. Virol. 2, 1069 (2020).

12. Sharma, G., Hanania, N. A. \& Shim, Y. M. The aging immune system and its relationship to the development of chronic obstructive pulmonary disease. Proc. Am. Thorac. Soc. 6, 573-580 (2009).

13. Saha, A. et al. Tocilizumab: a therapeutic option for the treatment of cytokine storm syndrome in COVID-19. Arch. Med. Res. 51, 595-597 (2020).

14. Bhattacharya, M. et al. Development of epitope-based peptide vaccine against novel coronavirus 2019 (SARS-COV-2): Immunoinformatics approach. J. Med. Virol. 92, 618-631 (2020).

15. Blanco-Melo, D. et al. Imbalanced host response to SARS-CoV-2 drives development of COVID-19. Cell 181, 1036-1045. e1039 (2020).

16. Zhou, Z. et al. Overly exuberant innate immune response to SARS-CoV-2 infection. (2020).

17. Xiong, Y. et al. Transcriptomic characteristics of bronchoalveolar lavage fluid and peripheral blood mononuclear cells in COVID19 patients. Emerging microbes \& infections 9, 761-770 (2020).

18. Prasad, K. et al. Targeting hub genes and pathways of innate immune response in COVID-19: a network biology perspective. Int. J. Biol. Macromol. 163, 1-8 (2020).

19. Zakeri, N. S. S., Pashazadeh, S. \& MotieGhader, H. Gene biomarker discovery at different stages of Alzheimer using gene coexpression network approach. Sci. Rep. 10, 1-13 (2020).

20. Adhami, M., MotieGhader, H., Haghdoost, A. A., Afshar, R. M. \& Sadeghi, B. Gene co-expression network approach for predicting prognostic microRNA biomarkers in different subtypes of breast cancer. Genomics 112, 135-143 (2020).

21. Adhami, M., Sadeghi, B., Rezapour, A., Haghdoost, A. A. \& MotieGhader, H. Repurposing novel therapeutic candidate drugs for coronavirus disease-19 based on protein-protein interaction network analysis. BMC Biotechnol. 21, 1-11 (2021).

22. Sardar, R., Satish, D., Birla, S. \& Gupta, D. Comparative analyses of SAR-CoV2 genomes from different geographical locations and other coronavirus family genomes reveals unique features potentially consequential to host-virus interaction and pathogenesis. BioRxiv (2020).

23. Motieghader, H., Kouhsar, M., Najafi, A., Sadeghi, B. \& Masoudi-Nejad, A. mRNA-miRNA bipartite network reconstruction to predict prognostic module biomarkers in colorectal cancer stage differentiation. Mol. BioSyst. 13, 2168-2180 (2017).

24. MotieGhader, H., Masoudi-Sobhanzadeh, Y., Ashtiani, S. H. \& Masoudi-Nejad, A. mRNA and microRNA selection for breast cancer molecular subtype stratification using meta-heuristic based algorithms. Genomics 112, 3207-3217 (2020).

25. Rudrapal, M., Khairnar, J. \& Jadhav, G. Drug repurposing (DR): an emerging approach in drug discovery. Drug Repurposing Hypothesis Mol. Asp. Ther. Appl (2020).

26. Pushpakom, S. et al. Drug repurposing: progress, challenges and recommendations. Nat. Rev. Drug Discovery 18, 41-58 (2019).

27. Conte, F. et al. A paradigm shift in medicine: A comprehensive review of network-based approaches. Biochimica et Biophysica Acta (BBA)-Gene Regulatory Mechanisms 1863, 194416 (2020).

28. Fiscon, G., Conte, F., Farina, L. \& Paci, P. SAveRUNNER: a network-based algorithm for drug repurposing and its application to COVID-19. PLoS Comput. Biol. 17, e1008686 (2021).

29. Fiscon, G. \& Paci, P. SAveRUNNER: an R-based tool for drug repurposing. BMC Bioinf. 22, 1-10 (2021).

30. Fiscon, G., Conte, F., Amadio, S., Volonté, C. \& Paci, P. Drug repurposing: a network-based approach to amyotrophic lateral sclerosis. Neurotherapeutics, 1-14 (2021).

31. Sibilio, P. et al.. In silico drug repurposing in COVID-19: a network-based analysis. Biomed. Pharmacother. 142, 111954 (2021).

32. Gysi, D.M. et al.. Network medicine framework for identifying drug-repurposing opportunities for COVID-19. Proc. Natl. Acad. Sci. 118 (2021).

33. Taz, T. A. et al. Network-based identification genetic effect of SARS-CoV-2 infections to Idiopathic pulmonary fibrosis (IPF) patients. Brief. Bioinf. 22, 1254-1266 (2021).

34. Zhou, Y. et al. Network-based drug repurposing for novel coronavirus 2019-nCoV/SARS-CoV-2. Cell Discov. 6, 1-18 (2020).

35. Du, H., Chen, F., Liu, H. \& Hong, P. Network-based virus-host interaction prediction with application to SARS-CoV-2. Patterns 2, 100242 (2021).

36. Sadegh, S. et al. Exploring the SARS-CoV-2 virus-host-drug interactome for drug repurposing. Nat. Commun. 11, 1-9 (2020).

37. Chen, Z. et al. Construction of an autophagy interaction network based on competitive endogenous RNA reveals the key pathways and central genes of SARS-CoV-2 infection in vivo. Microb. Pathogenesis, 105051 (2021).

38. Jensen, L. J. et al. STRING 8-a global view on proteins and their functional interactions in 630 organisms. Nucleic Acids Res. 37, D412-D416 (2009).

39. Han, H. et al. TRRUST v2: an expanded reference database of human and mouse transcriptional regulatory interactions. Nucleic Acids Res. 46, D380-D386 (2018).

40. Dweep, H. \& Gretz, N. miRWalk2.0: a comprehensive atlas of microRNA-target interactions. Nat. Methods 12, 697-697 (2015).

41. Freshour, S.L. et al.. Integration of the Drug-Gene Interaction Database (DGIdb 4.0) with open crowdsource efforts. Nucleic Acids Res. 49, D1144-D1151 (2021).

42. Wang, J., Lu, M., Qiu, C. \& Cui, Q. TransmiR: a transcription factor-microRNA regulation database. Nucleic Acids Res. 38, D119-D122 (2010).

43. Shannon, P. et al. Cytoscape: a software environment for integrated models of biomolecular interaction networks. Genome Res. 13, 2498-2504 (2003).

44. Patten, J. et al.. Multidose evaluation of 6,710 drug repurposing library identifies potent SARS-CoV-2 infection inhibitors In Vitro and In Vivo. bioRxiv (2021).

45. Choi, K.H., Shin, C.H., Lee, W.J., Ji, H. \& Kim, H.H. Dual-strand tumor suppressor miR-193b-3p and-5p inhibit malignant phenotypes of lung cancer by suppressing their common targets. Biosci. Rep. 39 (2019).

46. Hu, H., Li, S., Liu, J. \& Ni, B. MicroRNA-193b modulates proliferation, migration, and invasion of non-small cell lung cancer cells. Acta Biochim Biophys. Sin 44, 424-430 (2012).

47. Sun, L. et al. Regulation of RAB22A by mir-193b inhibits breast cancer growth and metastasis mediated by exosomes. Int. J. Oncol. 53, 2705-2714 (2018).

48. Hulin, J.-A. et al. MiR-193b regulates breast cancer cell migration and vasculogenic mimicry by targeting dimethylarginine dimethylaminohydrolase 1. Sci. Rep. 7, 1-15 (2017).

49. Hu, S. et al. CD44v6 targeted by miR-193b-5p in the coding region modulates the migration and invasion of breast cancer cells. J. Cancer 11, 260 (2020). 
50. Hashemi, Z. S., Moghadam, M. F., Farokhimanesh, S., Rajabibazl, M. \& Sadroddiny, E. Inhibition of breast cancer metastasis by co-transfection of miR-31/193b-mimics. Iran. J. Basic Med. Sci. 21, 427 (2018).

51. Filipska, M. et al. MiR-192 and miR-662 enhance chemoresistance and invasiveness of squamous cell lung carcinoma. Lung Cancer 118, 111-118 (2018).

52. Zou, P. et al. miR-192-5p suppresses the progression of lung cancer bone metastasis by targeting TRIM44. Sci. Rep. 9, 1-9 (2019).

53. Wang, T., Li, W., Li, H. \& Li, W. Dysregulation of exosomal miR-192 and miR-194 expression in lung adenocarcinoma patients. Saudi J. Biol. Sci. 28, 1561-1568 (2021).

54. Zhang, F. et al.. MiR-192 confers cisplatin resistance by targeting Bim in lung cancer. Zhongguo fei ai za zhi 17 (2014).

55. Shang, G. et al. MicroRNA-192 inhibits the proliferation, migration and invasion of osteosarcoma cells and promotes apoptosis by targeting matrix metalloproteinase-11. Oncol. Lett. 15, 7265-7272 (2018).

56. Cai, X. et al. miR-215 suppresses proliferation and migration of non-small cell lung cancer cells. Oncol. Lett. 13, 2349-2353 (2017).

57. Yao, Y., Shen, H., Zhou, Y., Yang, Z. \& Hu, T. MicroRNA-215 suppresses the proliferation, migration and invasion of non-small cell lung carcinoma cells through the downregulation of matrix metalloproteinase-16 expression. Exp. Ther. Med. 15, 3239-3246 (2018).

58. Hou, Y. et al. miR-215 functions as a tumor suppressor and directly targets ZEB2 in human non-small cell lung cancer. Oncol. Lett. 10, 1985-1992 (2015).

59. Centa, A. et al. Deregulated miRNA expression is associated with endothelial dysfunction in post-mortem lung biopsies of COVID-19 patients. Am. J. Physiol. Lung Cell. Mol. Physiol. 320, L405-L412 (2021).

60. Hart, M. et al. Identification of miR-34a-target interactions by a combined network based and experimental approach. Oncotarget 7, 34288 (2016).

61. Aida, R. et al. miR-34a-5p might have an important role for inducing apoptosis by down-regulation of SNAI1 in apigenin-treated lung cancer cells. Mol. Biol. Rep. 48, 2291-2297 (2021).

62. Kim, W. R. et al. Expression Analyses of MicroRNAs in Hamster Lung Tissues Infected by SARS-CoV-2. Mol. Cells 43, 953 (2020).

63. Wicik, Z. et al. ACE2 interaction networks in COVID-19: a physiological framework for prediction of outcome in patients with cardiovascular risk factors. J. Clin. Med. 9, 3743 (2020).

64. Mariconti, M. et al. Correction to: Role of microRNAs in host defense against Echinococcus granulosus infection: a preliminary assessment. Immunol. Res. 67, 98-98 (2019).

65. Biswas, S., Haleyurgirisetty, M., Lee, S., Hewlett, I. \& Devadas, K. Development and validation of plasma miRNA biomarker signature panel for the detection of early HIV-1 infection. EBioMedicine 43, 307-316 (2019).

66. Ketprasit, N. et al. The characterization of extracellular vesicles-derived microRNAs in Thai malaria patients. Malar. J. 19, 1-14 (2020).

67. Wei, J. et al. MicroRNA-16 inhibits the proliferation and metastasis of human lung cancer cells by modulating the expression of YAP1. J. BU ON. Off. J. Balkan Union Oncol. 25, 862-868 (2020).

68. Paniri, A., Hosseini, M.M., Moballegh-Eslam, M. \& Akhavan-Niaki, H. Comprehensive in silico identification of impacts of ACE2 SNPs on COVID-19 susceptibility in different populations. Gene Rep. 22, 100979 (2021).

69. Teodori, L. et al.. MicroRNAs bioinformatics analyses identifying HDAC pathway as a putative target for existing anti-COVID-19 therapeutics. Front. Pharmacol. 11 (2020).

70. Gao, Y. \& Yang, F. MiR-26b regulates invasion and migration of lung cancer cells through targeting hENT1 depending on RhoA/ ROCK-1 pathway. Zhong nan da xue xue bao. Yi xue ban Journal of Central South University. Medical Sciences 42, 755-761 (2017).

71. Jiang, M., Li, X., Quan, X., Li, X. \& Zhou, B. MiR-92a family: a novel diagnostic biomarker and potential therapeutic target in human cancers. Front. Mol. Biosci. 6, 98 (2019)

72. Ren, P., Gong, F., Zhang, Y., Jiang, J. \& Zhang, H. MicroRNA-92a promotes growth, metastasis, and chemoresistance in nonsmall cell lung cancer cells by targeting PTEN. Tumor. Biol. 37, 3215-3225 (2016).

73. Yu, Y. et al. Plasma miR-92a-2 as a biomarker for small cell lung cancer. Cancer Biomark. 18, 319-327 (2017).

74. Gong, J. et al. The relationship between miR-17-5p, miR-92a, and let-7b expression with non-small cell lung cancer targeted drug resistance. J. Buon 22, 454-461 (2017).

75. Zhu, J. et al. CD73/NT5E is a target of miR-30a-5p and plays an important role in the pathogenesis of non-small cell lung cancer. Mol. Cancer 16, 1-15 (2017).

76. Guan, Y., Rao, Z. \& Chen, C. miR-30a suppresses lung cancer progression by targeting SIRT1. Oncotarget 9, 4924 (2018).

77. Quan, X., Li, X., Yin, Z., Ren, Y. \& Zhou, B. p53/miR-30a-5p/SOX4 feedback loop mediates cellular proliferation, apoptosis, and migration of non-small-cell lung cancer. J. Cell. Physiol. 234, 22884-22895 (2019).

78. Tang, R. et al. Downregulation of MiR-30a is associated with poor prognosis in lung cancer. Med. Sci. Monit. Int. Med. J. Exp. Clin. Res. 21, 2514 (2015).

79. Xu, X. et al. miR-30a-5p enhances paclitaxel sensitivity in non-small cell lung cancer through targeting BCL-2 expression. J. Mol. Med. 95, 861-871 (2017).

80. Xiao, H. MiR-7-5p suppresses tumor metastasis of non-small cell lung cancer by targeting NOVA2. Cell. Mol. Biol. Lett. 24, 1-13 (2019).

81. Lundstrom, K. (Multidisciplinary Digital Publishing Institute, 2020).

82. Yao, W. et al. The CDR1as/miR-7/TGFBR2 axis modulates EMT in silica-induced pulmonary fibrosis. Toxicol. Sci. 166, 465-478 (2018).

83. Zhang, X., Yang, D. \& Wei, Y. Overexpressed CDRlas functions as an oncogene to promote the tumor progression via miR-7 in non-small-cell lung cancer. Onco. Targets. Ther. 11, 3979 (2018).

84. Ahmad, S.I. 5-Fluorouracil in combination with deoxyribonucleosides and deoxyribose as possible therapeutic options for the Coronavirus, COVID-19 infection. Med. Hypotheses 142, 109754 (2020).

85. Khan, M.A. et al.. Comparative molecular investigation of the potential inhibitors against SARS-CoV-2 main protease: a molecular docking study. J. Biomol. Struct. Dyn. 1-7 (2020).

86. Strich, J. R. et al. Fostamatinib inhibits neutrophils extracellular traps induced by COVID-19 patient plasma: a potential therapeutic. J. Infect. Dis. 223, 981-984 (2021).

87. Tabassum, N., Zhang, H. \& Stebbing, J. Repurposing fostamatinib to combat SARS-CoV-2-induced acute lung injury. Cell Rep. Med. 1, 100145 (2020).

88. Malimova, M. et al. A high content screen for mucin-1-reducing compounds identifies fostamatinib as a candidate for rapid repurposing for acute lung injury during the COVID-19 pandemic. bioRxiv (2020).

89. Apostolidis, S.A. et al. Signaling through Fc $\gamma$ RIIA and the C5a-C5aR pathway mediates platelet hyperactivation in COVID-19. bioRxiv (2021).

90. Liu, D.-Y. et al. Drug repurposing for COVID-19 treatment by integrating network pharmacology and transcriptomics. Pharmaceutics 13, 545 (2021).

91. Hoepel, W. et al. High titers and low fucosylation of early human anti-SARS-CoV-2 IgG promote inflammation by alveolar macrophages. Sci. Transl. Med. 13 (2021). 
92. Vergis, N. et al. Multi-arm Trial of Inflammatory Signal Inhibitors (MATIS) for hospitalised patients with mild or moderate COVID-19 pneumonia: a structured summary of a study protocol for a randomised controlled trial. Trials (2021).

93. Kindrachuk, J. et al. Antiviral potential of ERK/MAPK and PI3K/AKT/mTOR signaling modulation for Middle East respiratory syndrome coronavirus infection as identified by temporal kinome analysis. Antimicrob. Agents Chemother. 59, 1088-1099 (2015).

94. Tabari, M.A.K., Khoshhal, H., Tafazoli, A., Khandan, M. \& Bagheri, A. Applying computer simulations in battling with COVID19, using pre-analyzed molecular and chemical data to face the pandemic. Inf. Med. Unlocked 21, 100458 (2020).

95. Revannasiddaiah, S., Devadas, S.K., Palassery, R., Pant, N.K. \& Maka, V.V. A potential role for cyclophosphamide in the mitigation of acute respiratory distress syndrome among patients with SARS-CoV-2. Medi. Hypotheses 144, 109850 (2020).

96. Brocato, R. L. et al. Disruption of adaptive immunity enhances disease in SARS-CoV-2-infected Syrian hamsters. J. Virol. 94, e01683-e1620 (2020).

97. Othenin-Girard, A. et al. Multisystem inflammatory syndrome with refractory cardiogenic shock due to acute myocarditis and mononeuritis multiplex after SARS-CoV-2 infection in an adult. Swiss Med. Weekly 150 (2020).

98. Corso, C.R., de Oliveira, N.M.T. \& Maria-Ferreira, D. Susceptibility to SARS-CoV-2 infection in patients undergoing chemotherapy and radiation therapy. J. Infect. Public Health (2021).

99. Schaecher, S. R. et al. An immunosuppressed Syrian golden hamster model for SARS-CoV infection. Virology 380, 312-321 (2008).

100. Al-Rashedi, N.A., Munahi, M.G. \& AH ALObaidi, L. Prediction of potential inhibitors against SARS-CoV-2 endoribonuclease: RNA immunity sensing. J. Biomol. Struct. Dyn. 1-14 (2020).

101. Lokhande, K.B., Doiphode, S., Vyas, R. \& Swamy, K.V. Molecular docking and simulation studies on SARS-CoV-2 Mpro reveals Mitoxantrone, Leucovorin, Birinapant, and Dynasore as potent drugs against COVID-19. J. Biomol. Struct. Dyn. 1-12 (2020).

102. Safavi, F. \& Nath, A. Silencing of immune activation with methotrexate in patients with COVID-19. J. Neurol. Sci. 415 (2020).

103. Durairajb, S.K.S. JAK Inhibition with Methotrexate as Treatment for COVID-19 Is a Double-Edged Sword. (2020).

104. Schälter, F. et al. Does methotrexate influence COVID-19 infection? Case series and mechanistic data. Arthritis Res. Ther. 23, $1-6(2021)$.

105. Pindiprolu, S.K.S. \& Pindiprolu, S.H. Plausible mechanisms of Niclosamide as an antiviral agent against COVID-19. Medi. Hypotheses 140, 109765 (2020).

106. Backer, V. et al. A randomized, double-blind, placebo-controlled phase 1 trial of inhaled and intranasal niclosamide: a broad spectrum antiviral candidate for treatment of COVID-19. Lancet Reg. Health-Europe 4, 100084 (2021).

107. Yu, S. et al. Niclosamide-clay intercalate coated with nonionic polymer for enhanced bioavailability toward COVID-19 treatment. Polymers 13, 1044 (2021).

108. Kunzelmann, K. Getting hands on a drug for Covid-19: Inhaled and Intranasal Niclosamide. Lancet Reg. Health-Europe 4 (2021).

109. Hamizi, K., Aouidane, S. \& Belaaloui, G. Etoposide-based therapy for severe forms of COVID-19. Medi. Hypotheses 142, 109826 (2020).

110. Takami, A. Possible role of low-dose etoposide therapy for hemophagocytic lymphohistiocytosis by COVID-19. Int. J. Hematol. $112,122-124(2020)$

111. MB, M., LV, M. \& FR, M. Etoposide treatment adjunctive to immunosuppressants for critically ill COVID-19 patients. J. Infect. (2020).

112. Lovetrue, B. The AI-discovered aetiology of COVID-19 and rationale of the irinotecan+ etoposide combination therapy for critically ill COVID-19 patients. Med. Hypotheses 144, 110180 (2020).

113. Wang, J. et al. ClusterViz: a cytoscape APP for cluster analysis of biological network. IEEE/ACM Trans. Comput. Biol. Bioinf. 12, 815-822 (2014).

114. Chen, X., Peng, H. \& Yin, Z. (Hindawi, 2016).

115. Li, M.-H., Fu, S.-B. \& Xiao, H.-S. Genome-wide analysis of microRNA and mRNA expression signatures in cancer. Acta Pharmacol. Sin. 36, 1200-1211 (2015).

116. Mullany, L. E. et al. MicroRNA-transcription factor interactions and their combined effect on target gene expression in colon cancer cases. Genes Chromosom. Cancer 57, 192-202 (2018).

117. Sherman, B. T. \& Lempicki, R. A. Systematic and integrative analysis of large gene lists using DAVID bioinformatics resources. Nat. Protoc. 4, 44 (2009).

118. Huang, D. W., Sherman, B. T. \& Lempicki, R. A. Bioinformatics enrichment tools: paths toward the comprehensive functional analysis of large gene lists. Nucleic Acids Res. 37, 1-13 (2009).

119. Li, J. et al. TAM 2.0: tool for MicroRNA set analysis. Nucleic Acids Res. 46, W180-W185 (2018).

120. Chen, E. Y. et al. Enrichr: interactive and collaborative HTML5 gene list enrichment analysis tool. BMC Bioinf. 14, 1-14 (2013).

121. Lamb, J. et al.. The Connectivity Map: using gene-expression signatures to connect small molecules, genes, and disease. Science 313, 1929-1935 (2006).

\section{Author contributions}

H.M.G. wrote the main manuscript. H.M.G and A.R. performed the analyses. H.M.G. and E.S. reconstructed the networks. H.M.G., A.R. and F.F.A. interpreted the results and wrote the manuscript. H.M.G., R.A.I. analyzed the results. All authors reviewed the manuscript.

\section{Funding}

This work was supported by the Islamic Azad University, Tabriz branch.

\section{Competing interests}

The authors declare no competing interests.

\section{Additional information}

Supplementary Information The online version contains supplementary material available at https://doi.org/ 10.1038/s41598-021-01410-3.

Correspondence and requests for materials should be addressed to H.M.

Reprints and permissions information is available at www.nature.com/reprints.

Publisher's note Springer Nature remains neutral with regard to jurisdictional claims in published maps and institutional affiliations. 
(c) (i) Open Access This article is licensed under a Creative Commons Attribution 4.0 International cc) License, which permits use, sharing, adaptation, distribution and reproduction in any medium or format, as long as you give appropriate credit to the original author(s) and the source, provide a link to the Creative Commons licence, and indicate if changes were made. The images or other third party material in this article are included in the article's Creative Commons licence, unless indicated otherwise in a credit line to the material. If material is not included in the article's Creative Commons licence and your intended use is not permitted by statutory regulation or exceeds the permitted use, you will need to obtain permission directly from the copyright holder. To view a copy of this licence, visit http://creativecommons.org/licenses/by/4.0/.

(C) The Author(s) 2021 